\title{
A Waveguide Transverse Broad Wall Slot Radiating Between Baffles
}

\author{
Dich, Mikael; Rengarajan, S.R.
}

Published in:

Proc. of IEEE Antenna and Propagation Society International Symposium

Link to article, DOI:

10.1109/APS.1997.631868

Publication date:

1997

Document Version

Publisher's PDF, also known as Version of record

Link back to DTU Orbit

Citation (APA):

Dich, M., \& Rengarajan, S. R. (1997). A Waveguide Transverse Broad Wall Slot Radiating Between Baffles. In Proc. of IEEE Antenna and Propagation Society International Symposium (pp. 1438-1441). IEEE. https://doi.org/10.1109/APS.1997.631868

\section{General rights}

Copyright and moral rights for the publications made accessible in the public portal are retained by the authors and/or other copyright owners and it is a condition of accessing publications that users recognise and abide by the legal requirements associated with these rights.

- Users may download and print one copy of any publication from the public portal for the purpose of private study or research.

- You may not further distribute the material or use it for any profit-making activity or commercial gain

- You may freely distribute the URL identifying the publication in the public portal 


\title{
A Waveguide Transverse Broad Wall Slot Radiating Between Baffles
}

\author{
Mikael Dich* \\ Dept. of Electromagnetic Systems, Technical University of Denmark \\ Building 348, 2800 Lyngby, Denmark, Phone: +4545881444 , \\ Fax: +454593 1634, E-mail: midi@emi.dtu.dk \\ Sembiam R. Rengarajan \\ Dept. of Electrical and Computer Engineering, California State University \\ Northridge, California 91330-8346, Phone: +18186773571 , \\ Fax:+18186772140, E-mail: srengarajan@csun.edu
}

\begin{abstract}
An analysis of the self impedance of a waveguide-fed transverse slots radiating between baffles is presented. The region exterior to the slot is treated as a parallel plate (PP) waveguide which radiates into half space through an aperture in an infinite ground plane. The slot problem is analyzed using a set of integral equations which are solved numerically using the method of moments. The results can be used in previously published design procedures for waveguidefed series slot arrays. The theoretical results will be validated using network measurements.
\end{abstract}

\section{Introduction}

The use of resonant transverse slot in the broad wall of a rectangular waveguide has been limited by the fact that the possible variation of its excitation is small and since no simple mechanism such as alternation of slot offset exists for the reversal of the phase of the slot excitation. This means that for broadside arrays the slot spacing must be chosen equal to one guide wavelength, resulting in severe grating lobes. Josefsson [1] suggests the use of baffles for the suppression of these lobes. In his novel paper he uses the method of moments to calculate the self impedance of the transverse slot in the case of two exterior regions, one being half space and the other being an infinite PP waveguide. In this paper we will analyze the self impedance of a transverse slot radiating between baffles of finite length. The wall thickness of the waveguide carrying the slot is included in the analysis. The results from the analysis can be used in the classical design procedure described in [2].

0-7803-4178-3/97/\$10.00 (C) 1997 IEEE 


\section{Method of Analysis}

Fig. 1 shows the geometry of the slot problem to be analyzed. It is assumed that the waveguide and baffle structure possess no geometrical variation in the direction of the $z$-axis so that the region bounded by the baffles and the waveguide can be assumed cylindrical. It is further assumed that a driving $\mathrm{TE}_{10}$ wave, traveling in the positive $z$-direction inside the waveguide, is incident on the slot.

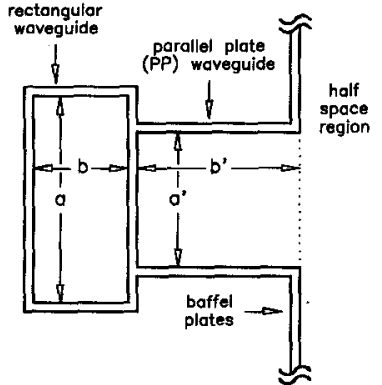

(a) side view

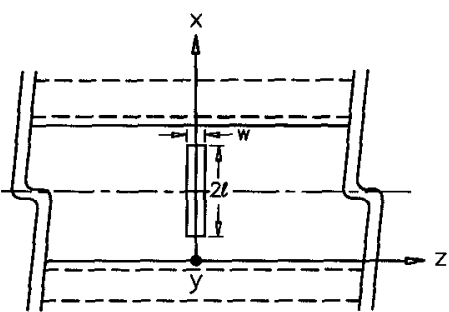

(b) front view

Figure 1: Geometry of a transverse broad wall slot radiating between baffles.

For the analysis of the slot problem we first divided the configuration into three regions. The three regions are: 1) the waveguide carrying the slot, 2) the interior of the slot and 3) the exterior region bounded by the baffles. The interaction between the fields in region 1 and 2 and between region 2 and 3 are modeled by two apertures at the ends of the waveguide that forms the slot. Using the field equivalence principles we can cover the two apertures with a thin sheet of conducting material and replace the tangential components of the electric fields in the apertures with equivalent magnetic currents. For the aperture that connects region 1 and 2 we use a magnetic current denoted by $\mathbf{M}_{12}$. For the aperture that connects region 2 and 3 we use a current denoted by $\mathbf{M}_{23}$.

Using these equivalent magnetic currents the continuity of the tangential components of the electric fields are satisfied across the apertures. To uniquely solve the slot problem we must also require that the tangential components of the magnetic fields are continuous:

$$
\begin{aligned}
\mathbf{H}_{i n c}+\mathbf{H}_{1}\left(-\mathbf{M}_{12}\right) & =\mathbf{H}_{2}\left(\mathbf{M}_{12}\right)+\mathbf{H}_{2}\left(-\mathbf{M}_{23}\right) \\
\mathbf{H}_{2}\left(\mathbf{M}_{12}\right)+\mathbf{H}_{2}\left(-\mathbf{M}_{23}\right) & =\mathbf{H}_{3}\left(\mathbf{M}_{23}\right)
\end{aligned}
$$

where $\mathbf{H}_{i n c}$ is the incident field in the waveguide when the apertures are shorted out with the conducting sheets and $\mathbf{H}_{m}\left(\mathbf{M}_{m n}\right)$ is the magnetic field in region $m$ scattered off the magnetic current which is equivalent to the tangential part of the electric field in the aperture that separates region $m$ and $n$. Eqs. (1) 
and (2) constitutes two coupled integral equations which we will solve using the method of moments.

For the expansion of the magnetic currents we will use the equivalent magnetic current of the electric field of the $\mathrm{TE}_{p 0}$ mode in the waveguide that forms the slot, i.e.

$$
\mathbf{M}_{p}(\zeta, \xi)=\widehat{\mathbf{x}} \cdot \sin \left(\frac{p \pi(\zeta+l)}{2 l}\right)
$$

where $\zeta$ and $\xi$ are the longitudinal and transverse slot coordinates localized to the center of slot. Employing a Galerkin type testing of the integral equations by dot-multiplying (1) and (2) with $\mathbf{M}_{q}$ given in (3) and by integrating over the respective apertures we obtain a set of linear equations for the coefficient in the expansion of the two magnetic currents $\mathbf{M}_{12}$ and $\mathbf{M}_{23}$.

The main task in the calculation of the terms in the linear equation system is the evaluation of the magnetic fields scattered off the magnetic currents. Inside the waveguide (region 1) a Green's function formulation similar to the one used in [1] has been employed. Inside the slot (region 2) the determination of the magnetic field becomes simple since each of the expansion functions $\mathbf{M}_{p}$ only couple to one waveguide mode of the waveguide that constitutes the slot. In the exterior region bounded by the baffles (region 3) one has first to solve the problem of the slot aperture radiating into a region constructed of a $\mathrm{PP}$ waveguide section of finite length connected to an infinite ground plane. This problem is solved using an integral equation for the magnetic field and where the unknown is a magnetic current equivalent to the tangential component of the electric field in the aperture that connects the PP section with half space.

When the coefficients to the expansion of the two magnetic currents $\mathbf{M}_{12}$ and $\mathrm{M}_{23}$ are found, the normalized self impedance of the slot is calculated as

$$
Z_{\text {selj }} / Z_{0}=-\frac{2 H_{\text {refl }}}{H_{\text {inc }}+\overline{H_{\text {scat }}}}
$$

where $H_{i n c}$ is the excitation of the incident $\mathrm{TE}_{10}$ wave $\mathbf{H}_{i n c}$ and $H_{\text {refl }}$ is the excitation of the $T E_{10}$ mode term of the magnetic field $\mathbf{H}_{1}\left(-\mathbf{M}_{12}\right)$ scattered off the slot inside the waveguide in the negative $z$-direction.

\section{Results}

All the following pertain to the computed values of the normalized self impedance $Z_{\text {self }} / Z_{0}$ of a transverse broad wall slot in a C-band waveguide with the internal dimensions $a \times b=47.55 \times 22.15 \mathrm{~mm}^{2}$. The dimensions of the slot are $2 l \times w=27 \times 2.5 \mathrm{~mm}^{2}$ and the wall thickness is $t=1.63 \mathrm{~mm}$. The dimensions of the baffles are $a^{\prime} \times b^{\prime}=32 \times 40 \mathrm{~mm}^{2}$. In Fig. 2 the real and imaginary part of the normalized self impedance is plotted as a function of the frequency. The two solid curves represent the computed self impedance when only one expansion. 
function has been used in each of the two apertures. In the case of the two dashed curves the expansion functions $\mathbf{M}_{\mathbf{1}} \cdots \mathbf{M}_{13}$ has been used. However, due to the symmetry of the slot configuration only expansion functions with an odd index is excited, giving a total of seven significant expansion functions.

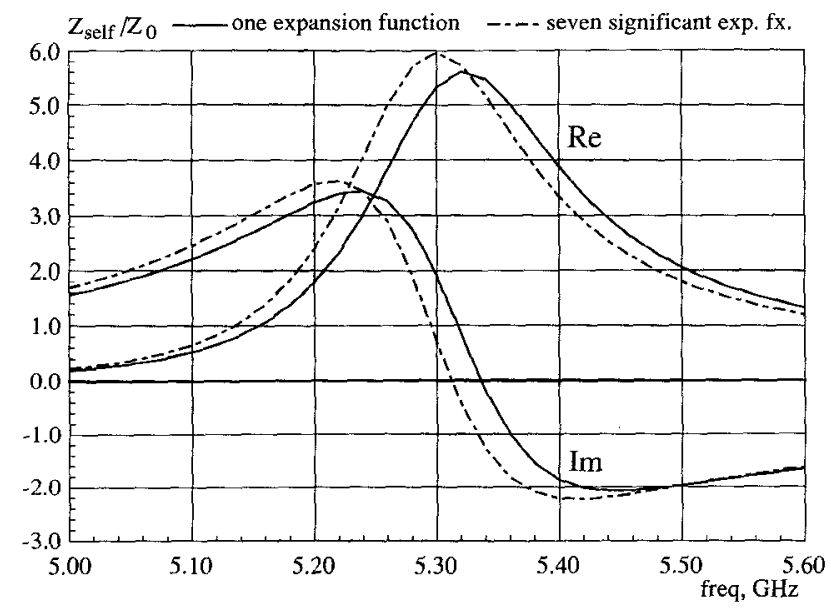

Figure 2: Real and imaginary parts of the normalized self impedance as a function of the frequency.

\section{Conclusion}

We have presented an analysis of the self impedance of a transverse broad wall slot radiating between baffles. At the time of writing the theoretical analysis has been completed and an antenna consisting of a single slot radiating between baffes is under construction. Comparisons between computed and measured values of the self impedance and the radiation pattern will be presented at the symposium.

\section{References}

[1] L. Josefsson, "A Waveguide Transverse Slot for Array Applications", IEEE Transactions on Antennas and Propagation, 41(7), pp. 845-850, July 1993.

[2] M. Orefice and R. S. Elliot, "Design of Waveguide-fed Series Slot Arrays", Proceedings of the IEEE, 129(4), pp. 165-169, August 1982. 